\title{
Impaired memory retrieval correlates with individual differences in cortisol response but not autonomic response
}

\author{
Tony W. Buchanan, ${ }^{1,3}$ Daniel Tranel, ${ }^{1}$ and Ralph Adolphs ${ }^{1,2}$ \\ ${ }^{1}$ Department of Neurology, University of lowa, lowa City, lowa 52242, USA; ${ }^{2}$ Division of Humanities \& Social Sciences and \\ Division of Biology, California Institute of Technology, Pasadena, California 91125, USA
}

\begin{abstract}
Stress can enhance or impair memory performance. Both cortisol release and sympathetic nervous system responses have been implicated in these differential effects. Here we investigated how memory retrieval might be affected by stress-induced cortisol release, independently of sympathetic nervous system stress responses. Thirty-two healthy participants (16 women) learned emotionally arousing and neutral words. One hour later, half of the participants underwent a stressor (cold pressor test) and the other half, a control warm water exposure, both followed by a delayed free recall task. The stressed participants were split into those who did (responders, $N=8$ ) and those who did not (nonresponders, $N=6$ ) show a cortisol response. Both responders and nonresponders showed comparable sympathetic nervous system activity (skin conductance level) during the cold pressor. The cortisol responders recalled significantly fewer words compared to nonresponders, and compared to control participants; this effect was most pronounced for moderately arousing words (compared to highly arousing and neutral words). These results suggest that individual differences in cortisol reactivity affect memory retrieval performance, and help to explain the differential effects of stress on memory.
\end{abstract}

It is well known that stress affects memory. However, the direction of this effect can vary-some studies have shown that stress enhances memory performance, while others have shown a detrimental effect of stress on memory (McEwen and Sapolsky 1995; Lupien and Lepage 2001; Kim and Diamond 2002). The reasons for these different effects may have to do with the variable effects of stress interacting with the multifarious nature of memory. There is considerable work showing that stress or cortisol administration can enhance memory consolidation (Roozendaal 2000; Buchanan and Lovallo 2001; Cahill et al. 2003). Specifically, this work has shown that emotionally arousing items receive more of a mnemonic benefit from post-learning glucocorticoids than do neutral items (Okuda et al. 2004; although others have reported no specific effect on emotional memory; e.g., Abercrombie et al. 2003). Conversely, research has shown that memory retrieval is impaired by stress or cortisol administration (de Quervain et al. 1998, 2000; Kuhlmann et al. 2005a,b). In a recent meta-analysis, Het et al. (2005) concluded that cortisol administration before retrieval exerted the greatest influence on memory performance compared to other administration regimes. Studies in animals and humans have suggested that the retrieval of emotional memories (as compared to neutral ones) may be most affected by stress and glucocorticoids (Roozendaal 2002; Kuhlmann et al. 2005a,b).

Just as there are differential effects of stress on various phases of memory, there are also large individual differences in how people respond to stress, as well as separable components of the stress response, which may influence how stress affects memory. Human stress research has elucidated the mechanisms that produce stress and especially stress-induced cortisol release. While the sympathetic nervous system component of the stress response is fairly easily elicited by many types of stressors, the

\footnotetext{
${ }^{3}$ Corresponding author.

E-mail tony-buchanan@uiowa.edu; fax (319) 356-4505.

Article and publication are at http://www.learnmem.org/cgi/doi/10.1101/ Im.206306.
}

release of cortisol is thought to depend on the elicitation of feelings of threat and uncontrollability (Dickerson and Kemeny 2004). The sympathetic and glucocorticoid components of the stress response may thus be differentiated. Tasks that are achievement-oriented may produce a sympathetic response without producing a cortisol response, while tasks involving negative affect and loss of control may produce both a sympathetic nervous system and cortisol response (Lovallo et al. 1985; Lovallo 2005). In all tasks, however, there are individual differences in how participants respond to various stressors-some individuals produce a large cortisol response to minimally stressful situations, while others do not show a cortisol response despite the stress researchers' best efforts to intensify the stressor.

These individual differences may be exploited to better understand the effects of stress on memory. If some individuals show a pronounced cortisol response to a task, while others respond with sympathetic changes (e.g., increased skin conductance) without showing a cortisol response, it should be possible to examine the effects of stress-induced cortisol on memory performance while controlling for the effects of sympathetic nervous system activity. This strategy has recently been applied to study the effects of stress on working memory. Elzinga and Roelofs (2005) demonstrated that subjects who responded to a public speaking task with significant cortisol and sympathetic responses showed reduced working memory performance. However, this working memory impairment was not found in participants who merely showed the sympathetic component of the stress response.

The present study was designed to examine the effects of stress-induced sympathetic and cortisol responses on memory retrieval. Based on the literature reviewed above, we predicted that individuals showing both a cortisol and sympathetic response to stress would show reduced memory retrieval, while those producing only a sympathetic response would not show this effect (i.e., memory would be unaffected). Additionally, we investigated how this stress effect would interact with the emo- 
tional nature of the memory material, by using emotional and neutral words as to-be-remembered stimuli. Previous work on the psychology and neurobiology of emotion has shown that highly arousing materials are processed differently from stimuli that are merely unpleasant (Kensinger and Corkin 2004; Buchanan et al. 2006b). We chose to address this issue by including stimuli that varied on the dimension of arousal: We included words that were unpleasant and moderately arousing, along with highly arousing, taboo words. In line with previous work (Kuhlmann et al. $2005 \mathrm{~b}$ ), we predicted that retrieval of emotionally arousing stimuli would be most reduced by stress-induced cortisol; we had no specific predictions about differential effects of stress on moderately versus highly arousing stimuli, however.

\section{Results}

\section{Responses to task}

Figure 1 shows cortisol levels across the task conditions. These data were analyzed using a 2 Sex $\times 3$ Group $\times 3$ Time multivariate ANOVA. The responders showed higher cortisol levels after the water task compared to the nonresponders and control participants (a group by time interaction: $F_{4,52}=5.5, P=0.001$, $\left.\eta^{2}=0.3\right)$. Post hoc analysis shows that the groups were not significantly different at baseline ( $P s>0.2)$; however, the responders had higher cortisol at both post-task time points (compared to control: Ps $<0.01$; compared to nonresponders: Ps $<0.08)$. Between-subjects analysis of sex effects in cortisol response showed no difference in the cortisol levels of men and women across the samples $\left(F_{1,24}=2.6, P=0.12, \eta^{2}=0.1\right)$, nor was there an interaction between responder status and sex $\left(F_{4,46}=1.7, P=0.2\right.$, $\left.\eta^{2}=0.13\right)$, although there was very low sample size for this analysis.

Table 1 provides results from the questionnaires and the skin conductance data for the three groups. There was a trend toward a group difference in negative affect in response to the water task $\left(F_{2,27}=2.6, P=0.09, \eta^{2}=0.16\right)$, with the responders reporting higher negative affect after the water task compared to the other groups (based on a one-way ANOVA on mean changes in negative affect from before to after the cold pressor test). All groups reported less positive affect after the water task $\left(F_{2,27}=44\right.$, $\left.P<0.0001, \eta^{2}=0.6\right)$, but there was no group difference in positive affect over time $(F<1)$. Pain ratings from the McGill Pain Questionnaire did not differ between the responders and nonresponders $\left[t_{12}=1.7, P=0.12\right]$, although the responders showed slightly higher pain ratings.

There was a significant main effect of group on skin conductance level during the cold pressor $\left(F_{2,27}=3.95, P=0.031\right.$, $\eta^{2}=0.23$ ). Post hoc analysis showed that this group difference was accounted for by a greater skin conductance level in the

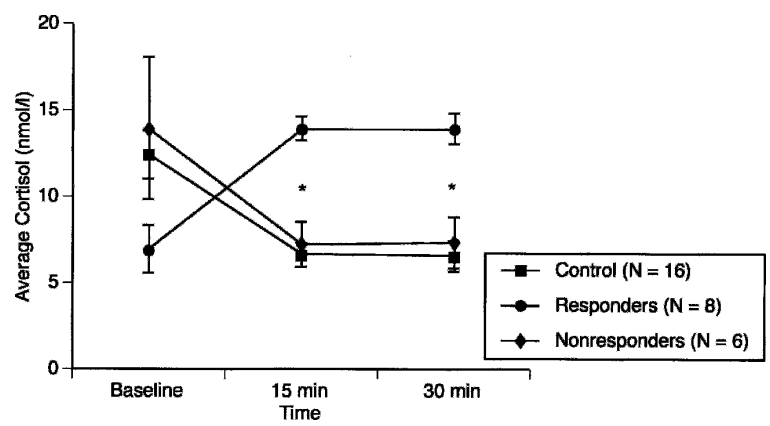

Figure 1. Cortisol levels throughout the experiment across groups. $\left(^{*}\right)$ A significant difference from control group ( $\left.P_{S}<0.01\right)$, and a marginally significant difference from nonresponders $(P s<0.08)$.
Table 1. Responses to cold pressor task and warm water task

\begin{tabular}{lccc}
\hline & $\begin{array}{c}\text { Controls } \\
(N=16)\end{array}$ & $\begin{array}{c}\text { Nonresponders } \\
(N=6)\end{array}$ & $\begin{array}{c}\text { Responders } \\
(N=8)\end{array}$ \\
\hline $\begin{array}{l}\text { Baseline negative } \\
\text { affect }\end{array}$ & $14.0 \pm 1.3$ & $16.2 \pm 2.1$ & $12.8 \pm 1.9$ \\
$\begin{array}{c}\text { Post-test negative } \\
\text { affect }\end{array}$ & $12.4 \pm 1.2$ & $14.2 \pm 1.9$ & $15.3 \pm 1.7$ \\
$\begin{array}{l}\text { Baseline positive affect } \\
\text { Post-test positive }\end{array}$ & $27.4 \pm 1.5$ & $28.7 \pm 2.5$ & $30.6 \pm 2.1$ \\
$\quad$ affect & $21.6 \pm 1.7$ & $23.8 \pm 2.7$ & $22.9 \pm 2.4$ \\
$\begin{array}{l}\text { McGill Pain } \\
\text { Questionnaire }\end{array}$ & $0.4 \pm 1.6$ & $14.3 \pm 2.6$ & $23.0 \pm 2.3$ \\
$\begin{array}{l}\text { Skin conductance } \\
\text { during task }\end{array}$ & $0.64 \pm 0.07$ & $1.1 \pm 0.14^{*}$ & $0.89 \pm 0.16$ \\
\begin{tabular}{l} 
Cortisol after task \\
\hline
\end{tabular} & $6.6 \pm 0.7$ & $7.2 \pm 1.3$ & $13.9 \pm 2.9^{* *}$ \\
\hline
\end{tabular}

(*) Significant difference from control group $(P<0.05)$; $\left({ }^{* *}\right)$ significant difference from both other groups $(P<0.05)$. Entries show mean \pm standard error of the mean. Skin conductance reported in microSiemens, cortisol reported in nanomole per liter $(\mathrm{nmol} / \mathrm{l})$.

nonresponder group compared to the control group $(P=0.038)$. The responders did not differ from the nonresponders in skin conductance level, however $(P>0.9)$. These data demonstrate that the two stress groups showed a comparable sympathetic response to the cold pressor, while showing divergent cortisol responses.

\section{Memory data}

Retrieval performance was expressed as the percentage of words recalled at delayed free recall in relation to the number recalled at immediate free recall. The proportions of words retrieved from each category were entered into a 2 Sex $\times 3$ Group (Controls, Responders, Nonresponders) $\times 3$ Word Category (Neutral, Moderate Arousal, High Arousal) multivariate ANOVA. There was no main effect of sex, nor were there interactions between sex and the other factors $(F<1)$. There was a significant effect of group $\left(F_{2,24}=3.9, P=0.03, \eta^{2}=0.25\right)$, with the responders recalling significantly fewer words compared to the nonresponders $(P=0.02)$ and the controls $(P=0.04)$ (see Fig. 2 and Table 2$)$. Additionally, there was a significant group-by-category interaction $\left(F_{3,40}=3.4\right.$, $P=0.022, \eta^{2}=0.22$ ). As shown in Figure 2, the responders recalled significantly fewer of the unpleasant words compared to both the nonresponders and controls $(P \mathrm{~s}<0.05)$. Interestingly, the nonresponders showed virtually equivalent (numerically slightly better) performance compared to controls across all word categories.

Correlation analyses were conducted among cortisol, skin conductance level, and percentage recall to examine the possible independent contributions of cortisol and sympathetic activity to memory performance. Across the whole participant sample, there was a significant negative correlation between cortisol level after the water task and percentage recall $(r=-0.4, P=0.03)$. There was no such association between skin conductance and recall $(r=-0.1, p>0.5)$. Skin conductance and cortisol after the task showed a modest positive correlation $(r=0.3, P=0.09)$. Correlation analyses were next conducted within participant groups (responders, nonresponders, and controls). Results show that only in the responder group was there a strong association between cortisol and memory retrieval $(r=-0.72, P=0.04)$; this was not true of the nonresponders $(r=-0.08)$ or the control group $(r=0.03)$. In both the responders and nonresponders, there were negative associations between skin conductance and memory retrieval ( $r=-0.4$ and $r=-0.67$, respectively); these associations did not reach statistical significance, however, because of the small sample sizes. The correlations between cortisol, skin conductance, and memory in the control group were not 
Table 2. Memory and rating data

\begin{tabular}{lccc}
\hline & $\begin{array}{c}\text { Controls } \\
(N=16)\end{array}$ & $\begin{array}{c}\text { Nonresponders } \\
(N=6)\end{array}$ & $\begin{array}{c}\text { Responders } \\
(N=8)\end{array}$ \\
\hline Immediate free recall & $19.1 \pm 1.2$ & $19.2 \pm 2.1$ & $17.5 \pm 0.7$ \\
Delayed free & & & \\
$\quad$ recall & $17.3 \pm 1.2$ & $18.3 \pm 2.2$ & $14.3 \pm 0.8$ \\
Recognition $\left(d^{\prime}\right)$ & $2.1 \pm 0.2$ & $2.3 \pm 0.3$ & $2.1 \pm 0.3$ \\
Valence rating & & & \\
$\quad$ Neutral & $3.1 \pm 0.06$ & $3.1 \pm 0.10$ & $3.1 \pm 0.09$ \\
$\quad$ Moderate & $1.3 \pm 0.08$ & $1.1 \pm 0.12$ & $1.4 \pm 0.11$ \\
$\quad$ High & $2.5 \pm 0.16$ & $1.9 \pm 0.26$ & $2.8 \pm 0.24^{*}$ \\
Arousal rating & $1.9 \pm 0.17$ & $1.8 \pm 0.28$ & $1.8 \pm 0.26$ \\
$\quad$ Neutral & $2.4 \pm 0.27$ & $2.0 \pm 0.44$ & $3.3 \pm 0.41$ \\
$\quad$ Moderate & $3.0 \pm 0.27$ & $2.6 \pm 0.44$ & $3.6 \pm 0.41$ \\
$\quad$ High & &
\end{tabular}

$\left(^{*}\right)$ Significant difference from nonresponder group $(P<0.05)$. Entries show mean \pm standard error of the mean.

significant $(r=0.34, P>0.2)$. These results demonstrate that even among those who show a cortisol response, there is a graded association, such that higher response is associated with reduced retrieval.

Recognition memory data were reduced by computing signal detection indices of performance. An index of discriminability $\left(d^{\prime}\right)$ (Snodgrass and Corwin 1988) is reported as a measure of recognition memory. One participant from the responder group did not complete the recognition test or the ratings (see Materials and Methods) because of computer error. There were no group differences in recognition of words $\left(F_{2,24}<1\right)$, nor was there a group-by-category interaction $\left(F_{4,46}<1.5\right)$. All groups recognized the high arousal words better than the other word categories $\left(F_{2,23}=13.6, P<0.0001, \eta^{2}=0.54\right)$. These data can be taken to show that stress affects retrieval mechanisms, but does not affect recognition performance.

\section{Word ratings}

Valence ratings of the words conformed to a priori classification, with unpleasant words rated more unpleasant than neutral and high arousal words (which were rated intermediate in valence; $\left.F_{2,23}=206, P<0.0001, \eta^{2}=0.95\right)$ (see Table 2 ). The groups rated the valence of the words slightly differently $\left(F_{2,24}=4.7\right.$, $\left.P=0.019, \eta^{2}=0.28\right)$, with the nonresponder group reporting lower valence ratings than the responders $(P=0.02)$ and the controls $(P=0.07)$. There was a trend toward an interaction between group and category $\left(F_{4,46}=2.1, P=0.1, \eta^{2}=0.15\right)$. Post hoc contrast tests showed that the responders rated the highly arousing words as more pleasant than the nonresponders $(P<0.05)$.

Ratings of arousal, similarly, conformed to expectations, with the high arousal word group receiving the highest arousal ratings, followed by the unpleasant words, which were rated higher than the neutral words $\left(F_{2,23}=10.4, P=0.001, \eta^{2}=0.47\right)$. There was a trend toward group difference in arousal ratings $\left(F_{2,24}=2.5, P=0.1, \eta^{2}=0.17\right)$, with the responders reporting higher arousal ratings than the other groups. There were no significant differences in the post hoc group comparisons, nor was there a group-by-category interaction in arousal ratings (see Table 2).

\section{Discussion}

Results from this study demonstrate that stress-induced cortisol release is associated with impaired memory retrieval, while stressful experience without the release of cortisol is not associated with this adverse effect on memory. Moreover, the effects of cortisol on memory retrieval were evident only for moderately arousing material. These results corroborate prior findings on the effects of stress and cortisol on memory performance, and extend those results by demonstrating individual differences in the effects of stress-induced cortisol on memory.

Animal research has demonstrated beneficial effects of stress on memory consolidation and deleterious effects on memory retrieval (Roozendaal 2002). Roozendaal has suggested that these disparate effects on cognition are dependent on actions of glucocorticoids acting via the basolateral amygdala (BLA) and hippocampus (Roozendaal 2002). Inactivation of the BLA blocks the enhanced memory consolidation (Roozendaal and McGaugh 1997) and the impaired memory retrieval (Roozendaal et al. 2003) induced by stress or glucocorticoid administration. These findings have been extended in human research by studies showing that consolidation of emotionally arousing stimuli is enhanced by post-learning stress (Cahill et al. 2003), whereas retrieval of emotionally arousing stimuli is impaired by preretrieval stress (Kuhlmann et al. 2005b). It is noteworthy that in the present study, the to-be-remembered stimuli that were most affected were the unpleasant/moderately arousing words and not the taboo/high arousing words or the neutral/low arousing words.

It is possible that the taboo/high arousal words were unaffected by stress manipulation because they were so highly arousing that the retrieval deficit did not affect the memory trace of these words. The moderately arousing words, in contrast, were less arousing on average and may achieve their memorable status through individual differences in physiological responses during encoding (Buchanan et al. 2006b). Considerable work has shown that interactions between the amygdala and hippocampus play a role in enhancing memory consolidation of emotional material (Buchanan and Adolphs 2004; Phelps 2004), and more recent work has suggested a role for the amygdala in retrieval of emotional material (Sharot et al. 2004; Buchanan et al. 2005, 2006a; Dolcos et al. 2005; Kensinger and Schacter 2005). It may be that cortisol released during stress may feed back to the level of the amygdala and hippocampus to impede optimal memory retrieval operations normally carried out by these structures.

Another possibility is that stress-induced cortisol affected retrieval operations of the prefrontal cortex (see also discussion of stress effects on working memory below). Kensinger and Corkin (2004) demonstrated that while memory for highly arousing words depended on the amygdala, memory for negatively valenced (and less arousing) words was dependent on prefrontal cortical activity. These authors also observed that memory for highly arousing words was not affected by an attentional modulation during encoding, suggesting that memory enhancement for these words occurs automatically even when attention is di-

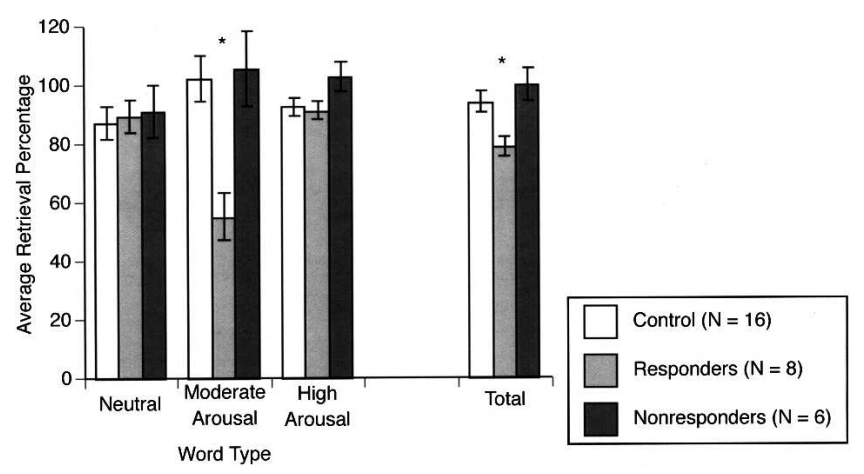

Figure 2. Effects of stress cortisol response and nonresponse on memory retrieval of words after a cold pressor test. Results are expressed as the percentage of immediate free recall from the neutral, moderate arousal, and high arousal word categories. (*) Significant difference from the other two groups. 
verted. Memory for the less arousing, negatively valenced words, in contrast, was impaired by the attentional diversion, suggesting that controlled processing is necessary for these words to receive a memory enhancement. Results from the present study demonstrate that recall of highly arousing words is not affected by stress-induced cortisol at retrieval, suggesting that memory for these words is unaffected by manipulations at encoding or retrieval. Memory for moderately arousing words, in contrast, is more susceptible to manipulations both at encoding and retrieval. The effect of stress on memory was only found for free recall performance and not for recognition. This finding has been reported previously (de Quervain et al. 2000; Kuhlmann et al. $2005 \mathrm{~b}$ ) and suggests that it is the more cognitively demanding act of recall that is affected and not more automatic processes such as the more simple visual recognition. Further research should more closely examine the neural correlates of stress effects on memory retrieval. We note that the present work offers only indirect evidence regarding the role of the amygdala or prefrontal cortex in retrieval of emotional memories, but the consistency in results between the present work and other research that has manipulated or measured neural activity is noteworthy.

The time course of the effect of stress on memory is a topic of much interest. The present results showed impaired memory retrieval caused by a stressor applied immediately before retrieval and only $1 \mathrm{~h}$ after encoding. Previous work has documented negative effects of stress or cortisol on memory performance on the same day (Kirschbaum et al. 1996; Newcomer et al. 1999), but in these studies, participants were stressed/administered cortisol before learning, thereby confounding the effects of treatment on encoding versus retrieval mechanisms. More recent work has carefully controlled for the timing of stress/cortisol treatment and shown that the retrieval phase is most adversely affected (de Quervain et al. 1998; de Quervain et al. 2000; Kuhlmann et al. 2005a,b), while stress or cortisol treatment after learning may actually facilitate memory when retrieval is tested under unstressed conditions (Cahill et al. 2003). The present study used the same cold pressor stress manipulation as Cahill et al. (2003), but it was applied $1 \mathrm{~h}$ after encoding, $10 \mathrm{~min}$ before retrieval. Cahill and colleagues, in contrast, administered the cold pressor immediately after encoding; memory retrieval was tested $1 \mathrm{wk}$ later under unstressed conditions. While our participants who produced a cortisol response showed reduced memory for emotionally arousing materials, those from the Cahill study showed enhanced memory for emotionally arousing material. These findings further highlight the importance of considering the phase of memory, the emotionality of the to-be-remembered items, and the timing of the stressor, when considering the effects of stress on memory.

Work from both animals and humans has shown that individual differences in physiological activity to stressful or novel situations can influence cognitive function (Tuinstra et al. 2000; McIntyre et al. 2002; Elzinga and Roelofs 2005). McIntyre and colleagues (McIntyre et al. 2002) measured norepinephrine (NE) levels in the amygdala using in vivo microdialysis while rats underwent inhibitory avoidance training footshock. Results showed substantial individual variability in intra-amygdala NE response (ranging from $100 \%$ to $>700 \%$ of baseline level). Interestingly, the NE response was strongly correlated with memory performance ( $r \mathrm{~s}>0.7) 1 \mathrm{~d}$ later. Assessing the effects of stress-induced cortisol and sympathetic responses on working memory performance in healthy humans, Elzinga and Roelofs (2005) recently demonstrated impaired working memory performance while performing a stress task during which both cortisol and sympathetic activity were elevated. Neither increased cortisol alone nor increased sympathetic activity alone led to impaired working memory; a combination of the two was required. This result is similar to our findings in the realm of declarative memory. Cortisol responders in the present study showed a strong negative correlation between post-stress cortisol and memory performance, while the association between sympathetic activity and memory was much smaller. An important caveat to our findings is that the responder group had lower pre-stress cortisol levels than the other groups (although this difference was not statistically significant). There has been considerable research documenting individual differences in cortisol levels and responses (Kudielka and Kirschbaum 2005; Lovallo 2005), and this difference brings up the possibility that these subjects differed from the other groups in more ways than merely cortisol response. The split into responders versus nonresponders based on differences other than those determined by the experimenters further limits our ability to ascribe differences in memory retrieval to cortisol response per se. Further research addressing the effects of personality, genetics, and life history on stress and memory should help to elucidate these effects.

In the present investigation and the study on working memory by Elzinga and Roelofs (2005), only those participants showing cortisol responses had lower cognitive performance. There is considerable evidence on the interactions of stress and the prefrontal cortex (Arnsten and Goldman-Rakic 1998; Sullivan and Gratton 1999, 2002; Roozendaal et al. 2004). Functional neuroimaging studies have shown that both working memory and declarative memory retrieval are associated with prefrontal cortical activity (Braver et al. 1997; Buckner and Wheeler 2001). The present results and those of Elzinga and Roelofs (2005) suggest that working memory and long-term memory retrieval mechanisms are similarly impaired in stressful situations involving the release of cortisol.

Previous work on stress, emotion, and memory has documented sex differences in performance (Wolf et al. 2001; Cahill and van Stegeren 2003). We did not find sex differences in memory performance in this study, although it should be noted that our responder group was comprised of 5 men and 3 women, a small sample size. The finding of more male than female cortisol responders to laboratory stress has been previously reported (Elzinga and Roelofs 2005). The inclusion of more participants, including more women responders, would better allow for testing sex differences in the effects we report here. A recent study has shown that administration of a high stress-level dose of cortisol to women results in memory retrieval impairment (Kuhlmann et al. 2005a). Higher cortisol levels, therefore, should result in similar patterns of cognitive effects in both men and women.

Results from this study further characterize the relationship between stress and memory. Cortisol response appears to be necessary for the negative effects of stress on retrieval. These results are similar to recent work from animals and humans examining declarative memory retrieval and working memory performance. Future work could more carefully address the underlying mechanisms that are affected by stress in order to better understand how stress affects neural processes and their associated cognitive products.

\section{Materials and Methods}

\section{Participants}

Thirty-two healthy volunteers (16 men, 16 women) between the ages of 18 and 21 (mean age: $18.9 \pm 0.8$ ) participated in the study for class credit. Participants were excluded from the study if they were taking any psychiatric, neurological, or corticosteroid-based medications. Participants were excluded if they reported working overnight shift work or if they had unusual sleep patterns. Two female participants in the stress condition had baseline cortisol values that were 2 SD above the mean of all 
other participants' data. These two participants' data were excluded, leaving a total $N$ of 30 (14 in the stress group and 16 in the control group). The study was approved by the Institutional Review Board of the University of Iowa; written informed consent was obtained from all participants.

\section{Materials}

A list of 80 words was compiled, including 40 emotionally neutral words (e.g., barrel, vehicle), 20 unpleasant moderately arousing words (e.g., killer, anger), and 20 high arousal words (e.g., masturbate, rape). The unpleasant emotional words and neutral words were drawn from the Affective Norms for English Words (ANEW) database (Bradley and Lang 1999). The unpleasant words were chosen because they were low on pleasantness (valence) and relatively high on arousal (mean normative rating of valence $=2.0$; arousal $=6.1$, on scales of $1-9)$. The neutral words were chosen to be in the middle range on pleasantness $(M=5.5)$ and low on arousal $(M=4.1)$. High arousal words were sexually explicit words including profanities (LaBar and Phelps 1998). These word categories were chosen to examine a wide range of arousing material, as arousal of to-be-remembered stimuli is highly predictive of subsequent memory (Bradley et al. 1992). Since most of the high arousal words are not included in traditional counts of word frequency (Francis and Kucera 1982), estimates of frequency were computed using the Alta Vista internet search engine (Blair et al. 2002). Each word was entered into the search function of Alta Vista; the number of hits returned was recorded as the frequency estimate for each word. Results of this analysis showed that there was not a significant difference in word frequency across the categories, $F_{3,76}=1.7, P=0.174$. The mean frequencies for each category were neutral: 4,617,834 \pm 686,574 (SEM); unpleasant/moderate arousal: 2,935,443 \pm 320,820; and high arousal: 6,242,050 $\pm 1,275,424$. (It should be acknowledged that the large number of sexually explicit internet Web sites might have overestimated the frequency of the high arousal words.) During encoding, each participant saw 20 neutral words, 10 unpleasant/moderate arousal words, and 10 high arousal words.

\section{Procedure}

Each participant reported to the laboratory between 1300 and $1500 \mathrm{~h}$ to control for the diurnal cycle of cortisol. After filling out the consent form, participants filled out the Positive Affectivity/ Negative Affectivity Schedule (PANAS) (Watson et al. 1988) to measure baseline positive and negative affect. Next, a baseline saliva sample was collected, and participants were connected to psychophysiological recording electrodes for measurement of skin conductance level. Each participant viewed a randomly selected set of 20 neutral, 10 unpleasant/moderate arousal, and 10 high arousal words drawn from the total list of 80 words. The remaining 40 words were used as foils in the recognition memory test (see description below). Two buffer words were presented at the beginning and end of the stimulus presentation to control for primacy and recency effects. These buffer words were not included in subsequent analyses of memory. Each word was presented for 4 sec with an interstimulus interval (blank screen) randomly ranging between 3000 and $3950 \mathrm{msec}$. Words were presented in 40-point white Arial font on a black background at the center of a 19-inch monitor. Stimuli were presented via Presentation software (Neurobehavioral Systems). Participants were asked to attend to the words and to try to remember as many of them as they could (explicit encoding).

Immediately after stimulus presentation, participants completed a free recall test, during which the participants wrote down as many words as they could recall on a blank sheet of paper. Participants were allowed 5 min for the completion of the free recall task. During the hour after stimulus presentation, participants worked on nonverbal distractor tasks. At 1-h postencoding, participants completed either a cold pressor or warm water control task. In the cold pressor task, participants submerged their right hand up to the wrist in ice water $\left(0^{\circ}-4^{\circ} \mathrm{C}\right)$. They were asked to maintain their hand in the water for $3 \mathrm{~min}$, although they were allowed to remove their hand if the pain became unbearable (two participants removed their hand from the cold water before 3 min had elapsed ${ }^{4}$ ). The cold pressor task is effective in producing sympathetic arousal and cortisol response in most participants (Lovallo 1975; Cahill et al. 2003). The warm water control task was identical to the cold pressor task, except that the water temperature was warm $\left(37^{\circ}-40^{\circ} \mathrm{C}\right)$. Immediately after the water task, participants were allowed to dry off their hand and were then asked to complete the PANAS again, and the McGill Pain Questionnaire (Melzack 1987). At 10 min post-water test, participants gave another saliva sample and then completed the delayed free recall task.

Participants then completed a recognition memory test; they viewed all 80 words ( 40 they had seen previously, and 40 new words) and were asked to respond "yes" or "no" as to whether they remembered seeing the word before. After responding to the recognition question, the participant rated each word on 5-point scales of pleasantness $(1=$ unpleasant; $5=$ very pleasant) and arousal ( $1=$ low arousal; $5=$ high arousal). After the recognition task, participants contributed a third and final saliva sample.

\section{Saliva assessment for cortisol measurement}

Saliva was collected using Salivette collection tubes (Sarstedt). As noted, three samples were collected: before the water test; $15 \mathrm{~min}$ after the water test; and 30 min after the water test. Samples were stored at $-20^{\circ} \mathrm{C}$ until assayed. Salivary cortisol was measured with a commercial immunoassay kit with chemiluminescence detection (CLIA; IBL Hamburg). The intra-assay and inter-assay coefficients of variation were $<10 \%$.

\section{Psychophysiological measures}

Skin conductance was measured using two $\mathrm{Ag}-\mathrm{AgCl}$ electrodes placed on the thenar and hypothenar surfaces of the left palm. Signals were recorded using a Biopac MP150 (Biopac Systems). Tonic skin conductance level was measured in microSiemens throughout the water task.

\section{Data management and analysis}

Because we were interested in comparing cortisol responders and nonresponders to control participants, participants in the cold pressor group were split into responders (those who had higher cortisol values after the cold pressor compared to before the cold pressor; $N=8,5$ men and 3 women) and nonresponders (those who had lower cortisol after the cold pressor compared to before the cold pressor; $N=6,3$ men and 3 women). These groups were compared to the control group $(N=16)$ in all subsequent analyses. Also, since previous research has shown sex differences in cortisol response to laboratory stress (Kudielka and Kirschbaum 2005) and in emotional memory (Cahill and van Stegeren 2003), we included sex as a factor in our analyses. Analyses include the $\eta^{2}$ measure of effect size where appropriate. Followup contrasts use the Bonferroni correction procedure to control for inflation of Type I error rate.

\section{Acknowledgments}

This study was supported by the following grants: MH067681 from National Institute of Mental Health and NS19632 from National Institute of Neurological Disorders and Stroke. The authors thank Clemens Kirschbaum for conducting the cortisol assays.

\section{References}

Abercrombie, H., Kalin, N.H., Thurow, M.E., Rosenkranz, M.A., and Davidson, R.J. 2003. Cortisol variation in humans affects memory for emotionally laden and neutral information. Behav. Neurosci. 117: $505-516$.

Arnsten, A.F.T. and Goldman-Rakic, P.S. 1998. Noise stress impairs prefrontal cortical cognitive function in monkeys: Evidence for a

${ }^{4}$ As it turned out, both of these participants were females in the responder group. 
hyperdopaminergic mechanism. Arch. Gen. Psychiatry 55: 362-369.

Blair, I.V., Urland, G.R., and Ma, J.E. 2002. Using Internet search engines to estimate word frequency. Behav. Res. Methods Instrum. Comput. 34: 286-290.

Bradley, M.M. and Lang, P.J. 1999. Affective norms for English words. NIMH Center for the Study of Emotion and Attention, Gainesville, FL.

Bradley, M.M., Greenwald, M.K., Petry, M.C., and Lang, P.J. 1992. Remembering pictures: Pleasure and arousal in memory. J. Exp. Psychol. Learn. Mem. Cogn. 18: 379-390.

Braver, T.S., Cohen, J.D., Nystrom, L.E., Jonides, J., Smith, E.E., and Noll, D.C. 1997. A parametric study of prefrontal cortex involvement in human working memory. Neuroimage 5: 49-62.

Buchanan, T.W. and Adolphs, R. 2004. The neuroanatomy of emotional memory in humans. In Memory and emotion (eds. D. Reisberg and P. Hertel), pp. 42-75. Oxford University Press, New York.

Buchanan, T.W. and Lovallo, W.R. 2001. Enhanced memory for emotional material following stress-level cortisol treatment in humans. Psychoneuroendocrinology 26: 307-317.

Buchanan, T.W., Tranel, D., and Adolphs, R. 2005. Emotional autobiographical memories in amnesic patients with medial temporal lobe damage. J. Neurosci. 25: 3151-3160.

. 2006a. Memories for emotional autobiographical events following unilateral damage to medial temporal lobe. Brain 129: $115-127$.

Buchanan, T.W., Etzel, J.A., Adolphs, R., and Tranel, D. 2006b. The influence of autonomic arousal and semantic relatedness on memory for emotional words. Int. J. Psychophysiol. (in press).

Buckner, R.L. and Wheeler, M.E. 2001. The cognitive neuroscience of remembering. Nat. Rev. Neurosci. 2: 624-634.

Cahill, L. and van Stegeren, A. 2003. Sex-related impairment of memory for emotional events with $\beta$-adrenergic blockade. Neurobiol. Learn. Mem. 79: $81-88$.

Cahill, L., Gorski, L., and Le, K. 2003. Enhanced human memory consolidation with post-learning stress: Interaction with the degree of arousal at encoding. Learn. Mem. 10: 270-274.

de Quervain, D.J., Roozendaal, B., and McGaugh, J.L. 1998. Stress and glucocorticoids impair retrieval of long-term spatial memory. Nature 394: 787-790.

de Quervain, D.J., Roozendaal, B., Nitsch, R.M., McGaugh, J.L., and Hock, C. 2000. Acute cortisone administration impairs retrieval of long-term declarative memory in humans. Nat. Neurosci. 3: 313-314.

Dickerson, S.S. and Kemeny, M.E. 2004. Acute stressors and cortisol responses: A theoretical integration and synthesis of laboratory research. Psychol. Bull. 130: 355-391.

Dolcos, R., LaBar, K.S., and Cabeza, R. 2005. Remembering one year later: Role of the amygdala and the medial temporal lobe memory system in retrieving emotional memories. Proc. Natl. Acad. Sci. 102: $2626-2631$.

Elzinga, B.M. and Roelofs, K. 2005. Cortisol-induced impairments of working memory require acute sympathetic activation. Behav. Neurosci. 119: 98-103.

Francis, W.N. and Kucera, H. 1982. Frequency analysis of English usage: Lexicon and grammar. Houghton Mifflin, Boston.

Het, S., Ramlow, G., and Wolf, O.T. 2005. A meta-analytic review of the effects of acute cortisol administration on human memory. Psychoneuroendocrinology 30: 771-784.

Kensinger, E.A. and Corkin, S. 2004. Two routes to emotional memory: Distinct neural processes for valence and arousal. Proc. Natl. Acad. Sci. 101: $3310-3315$.

Kensinger, E.A. and Schacter, D.L. 2005. Retrieving accurate and distorted memories: Neuroimaging evidence for effects of emotion. Neuroimage 27: 167-177.

Kim, J.J. and Diamond, D.M. 2002. The stressed hippocampus, synaptic plasticity and lost memories. Nat. Rev. Neurosci. 3: 453-462.

Kirschbaum, C., Wolf, O.T., May, M., Wippich, W., and Hellhammer, D.H. 1996. Stress- and treatment-induced elevations of cortisol levels associated with impaired declarative memory in healthy adults. Life Sci. 58: $1475-1483$.

Kudielka, B.M. and Kirschbaum, C. 2005. Sex differences in HPA axis responses to stress: A review. Biol. Psychol. 69: 113-132.

Kuhlmann, S., Kirschbaum, C., and Wolf, O.T. 2005a. Effects of oral cortisol treatment in healthy young women on memory retrieval of negative and neutral words. Neurobiol. Learn. Mem. 83: 158-162.

Kuhlmann, S., Piel, M., and Wolf, O.T. 2005b. Impaired memory retrieval after psychosocial stress in healthy young men. J. Neurosci.
25: 2977-2982.

LaBar, K.S. and Phelps, E.A. 1998. Arousal-mediated memory consolidation: Role of the medial temporal lobe in humans. Psychol. Sci. 9: 490-493.

Lovallo, W.R. 1975. The cold pressor test and autonomic function: A review and integration. Psychophysiology 12: 268-282.

- 2005. Stress and health: Biological and psychological interactions. Sage Publications, Thousand Oaks, CA.

Lovallo, W.R., Wilson, M.F., Pincomb, G.A., Edwards, G.L., Tompkins, P., and Brackett, D. 1985. Activation patterns to aversive stimulation in man: Passive exposure versus effort to control. Psychophysiology 22: $283-291$.

Lupien, S.J. and Lepage, M. 2001. Stress, memory, and the hippocampus: Can't live with it, can't live without it. Behav. Brain Res. 127: 137-158.

McEwen, B.S. and Sapolsky, R.M. 1995. Stress and cognitive function. Curr. Opin. Neurobiol. 5: 205-216.

McIntyre, C.K., Hatfield, T., and McGaugh, J.L. 2002. Norepinephrine levels in the amygdala following inhibitory avoidance training predict retention score. Eur. J. Neurosci. 16: 1223-1226.

Melzack, R. 1987. The short-form McGill Pain Questionnaire. Pain 30: $191-197$.

Newcomer, J.W., Selke, G., Melson, A.K., Hershey, T., Craft, S., Richards, K., and Alderson, A.L. 1999. Decreased memory performance in healthy humans induced by stress-level cortisol treatment. Arch. Gen. Psychiatry 56: 527-533.

Okuda, S., Roozendaal, B., and McGaugh, J.L. 2004. Glucocorticoid effects on object recognition memory require training-associated emotional arousal. Proc. Natl. Acad. Sci. 101: 853-858.

Phelps, E.A. 2004. Human emotion and memory: Interactions of the amygdala and hippocampal complex. Curr. Opin. Neurobiol. 14: $198-202$.

Roozendaal, B. 2000. Glucocorticoids and the regulation of memory consolidation. Psychoneuroendocrinology 25: 213-238.

- 2002. Stress and memory: Opposing effects of glucocorticoids on memory consolidation and retrieval. Neurobiol. Learn. Mem. 81: $150-154$.

Roozendaal, B. and McGaugh, J.L. 1997. Basolateral amygdala lesions block the memory-enhancing effect of glucocorticoid administration in the dorsal hippocampus of rats. Eur. J. Neurosci. 9: 76-83.

Roozendaal, B., Griffith, Q.K., Buranday, J., de Quervain, D.J.-F., and McGaugh, J.L. 2003. The hippocampus mediates glucocorticoid-induced impairment of spatial memory retrieval: Dependence on the basolateral amygdala. Proc. Natl. Acad. Sci. 100: $1328-1333$.

Roozendaal, B., McReynolds, J.R., and McGaugh, J.L. 2004. The basolateral amygdala interacts with the medial prefrontal cortex in regulating glucocorticoid effects on working memory impairment. $J$. Neurosci. 24: 1385-1392.

Sharot, T., Delgado, M.R., and Phelps, E.A. 2004. How emotion enhances the feeling of remembering. Nat. Neurosci. 7: 1376-1380.

Snodgrass, J.G. and Corwin, J. 1988. Pragmatics of measuring recognition memory: Applications to dementia and amnesia. J. Exp. Psychol. Gen. 117: 34-50.

Sullivan, R.M. and Gratton, A. 1999. Lateralized effects of medial prefrontal cortex lesions on neuroendocrine and autonomic stress responses in rats. J. Neurosci. 19: 2834-2840.

. 2002. Prefrontal cortical regulation of hypothalamic-pituitary-adrenal function in the rat and implications for psychopathology: Side matters. Psychoneuroendocrinology 27: $99-114$.

Tuinstra, T., Verheij, M., Willemen, A., Iking, J., Heeren, D.J., and Cools, A.R. 2000. Retrieval of spatial information in Nijmegen high and low responders: Involvement of $\beta$-adrenergic mechanisms in the nucleus accumbens. Behav. Neurosci. 114: 1088-1095.

Watson, D., Clark, L.A., and Tellegen, A. 1988. Development and validation of brief measures of positive and negative affect: The PANAS scales. J. Pers. Soc. Psychol. 54: 1063-1070.

Wolf, O.T., Schommer, N.C., Hellhammer, D.H., McEwen, B.S., and Kirschbaum, C. 2001. The relationship between stress induced cortisol levels and memory differs between men and women. Psychoneuroendocrinology 26: 711-720.

Received December 9, 2005; accepted in revised form February 28, 2006. 


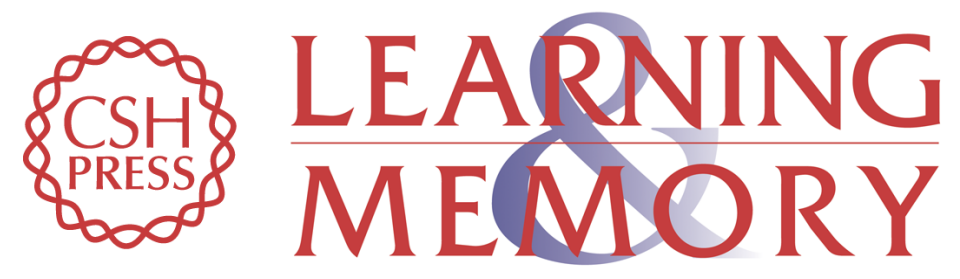

\section{Impaired memory retrieval correlates with individual differences in cortisol response but not autonomic response}

Tony W. Buchanan, Daniel Tranel and Ralph Adolphs

Learn. Mem. 2006, 13:

Access the most recent version at doi:10.1101//m.206306

References This article cites 45 articles, 9 of which can be accessed free at:

http://learnmem.cshlp.org/content/13/3/382.full.html\#ref-list-1

License

Email Alerting Receive free email alerts when new articles cite this article - sign up in the box at the Service top right corner of the article or click here. 